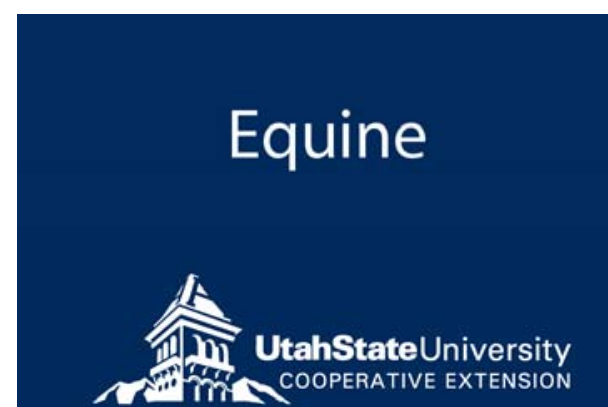

November 2013

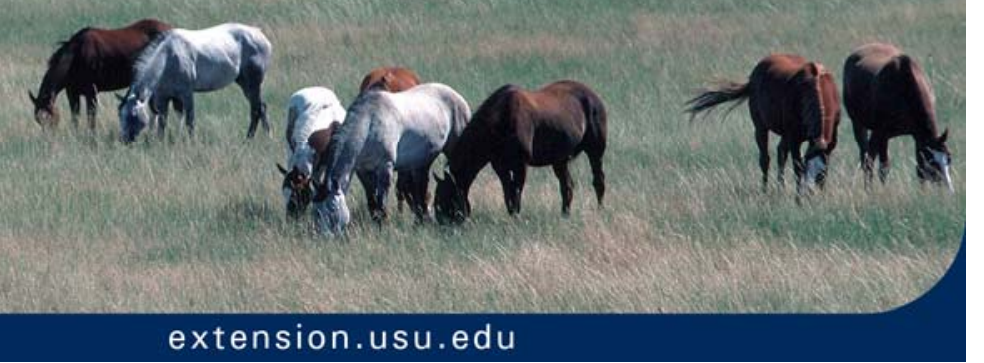

AG/Equine/2013-02pr

\title{
Equine Viral Arteritis (EVA)
}

Dirk K. Vanderwall, Associate Professor, Kerry A. Rood, Utah Extension Veterinarian, and Bruce L. King, Utah State Veterinarian

\section{Background}

Equine viral arteritis (EVA) has become a very important issue in the equine industry since the occurrence of a disease outbreak in the Quarter Horse breeding industry in 2006. Equine viral arteritis is a contagious disease syndrome caused by the Equine Arteritis Virus (EAV), which can infect all equines. Although EVA generally causes only mild upper respiratory tract disease in adult horses, it can cause fatal pneumonia in young foals and abortion in pregnant mares. In addition, stallions infected with EAV may become persistently infected sub-clinically (i.e., without evidence of infection) and shed infectious virus in their semen (i.e., become virus “carriers”). Transmission of the virus can occur via contact with respiratory secretions of clinically ill animals; contact with an aborted fetus and its associated fetal membranes/fluids; and/or contact with semen from a chronically infected stallion. Particularly important is the potential for widespread dissemination of the virus associated with the use of cooled and/or frozen semen (Figure 1) from chronically infected stallions, which was the principal mode of transmission in the Quarter Horse industry in 2006. That outbreak has lead to increased recognition of the disease, and prompted federal and state regulatory agencies and the breeding industry to take a heightened approach to control measures for EVA. Current recommendations focus on minimizing the risk of exposure that would lead to the introduction of the virus into susceptible groups of horses; vaccination of susceptible, “at-risk” horses (e.g., broodmares); and prevention of the disease in naive stallions in order to avoid the persistently infected carrier state.

\section{Clinical Signs}

In otherwise healthy adult horses, clinical signs of EAV infection may not be evident. When clinical signs do occur, they typically become evident 3 to 7 days post-exposure and may include: fever;

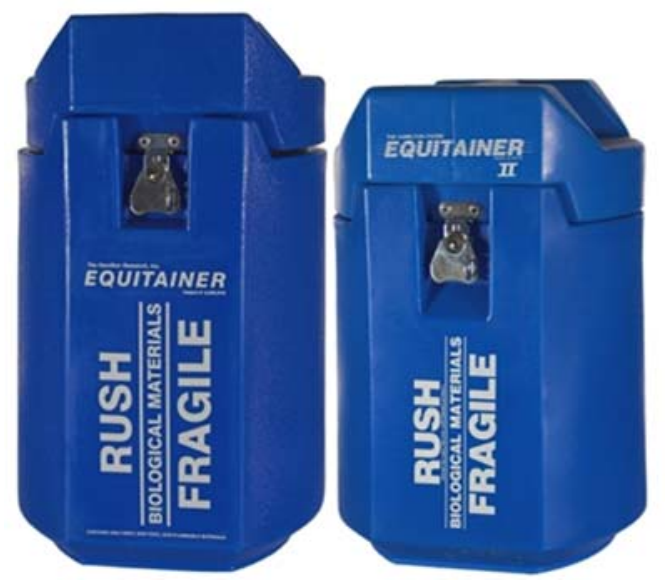

Figure 1. Typical cooled semen transport containers. Photo from Equitainer.

depression; anorexia; dependent edema of the limbs and ventral abdomen; urticaria; supra or periorbital edema; conjunctivitis; lacrimal discharge and serous to mucoid nasal discharge. Infection in unprotected pregnant mares frequently leads to abortion. Young foals can develop severe, life-threatening pneumonia. 


\section{Diagnosis}

Since the clinical signs of EVA are similar to other causes of respiratory diseases (e.g., influenza), EVA should be considered in any horse showing signs of respiratory disease. Similarly, EVA should be considered in any mare that aborts her pregnancy. Virus isolation can be attempted from swabs of nose, throat or eyes; or in the case of abortion, the aborted fetus, fetal fluids or placenta. The most common method of diagnosing EVA is by performing serology testing of blood for EAV neutralizing antibodies. Although the presence of neutralizing antibodies does not indicate active infection, it does indicate exposure to EAV has occurred. Extremely high antibody levels in a single sample or a four-fold or greater rise in antibody level in paired samples collected 14 to 28 days apart are generally indicative of recent active infection.

\section{Treatment}

There is no specific treatment of EVA, so efforts are directed at supportive care, such as rest and the use of anti-inflammatory medication to reduce a fever.

\section{Prevention and Control}

First and foremost, it is important to know the EAV status of resident stallions and/or stallions at other locations that will be used for breeding purposes (e.g., shipped or frozen semen). Serology can be used to assess prior exposure of a stallion to EAV; however, serology cannot distinguish between natural infection (that may have induced a "carrier" state in the stallion) or prior vaccination against EAV. Stallions that are seronegative (titer $<1: 4$ ) are not infective and mares can be bred to these stallions without risk of infection. Owners of seronegative stallions should strongly consider vaccinating the stallion to prevent the possibility of infection in the future with its attendant risk of development of the carrier state. Vaccination should be done at least 28 days prior to breeding/semen collection. Because serology cannot distinguish between natural infection and vaccination, it is imperative to have permanent documentation that a stallion was tested and shown to be seronegative prior to vaccination.

Seropositive stallions (titer $\geq 1: 4$ ), that have no record of having been seronegative prior to vaccination, should have their semen tested to determine if they are persistently infected. Testing involves examining an ejaculate of semen for the presence of EAV by the accredited laboratory at the Gluck Equine Research Center at the University of Kentucky (http://www2.ca.uky.edu/gluck/serveva.asp). Seropositive shedding stallions must be managed appropriately, which involves breeding only to vaccinated/seropositive mares and recognizing that all materials that come in contact with the stallion's semen are potentially infective. Vaccinated mares should be isolated for 21 days following their first breeding to a carrier stallion, because these mares may become transiently viremic with "wild-type" virus after breeding. Isolation for 21 days is not necessary after subsequent breeding, though isolation for at least 24 hours should be performed after every breeding to prevent mechanical spread of EAV if semen is voided from the mare's reproductive tract. Because of the severe health risk of EVA in young foals, breeding post-partum mares (even if vaccinated) to EAV shedding stallions should be avoided, because of the potential for transmission of the virus to the mare's foal.

It is important to note that vaccinating horses against EVA is done using a modified-live virus vaccine, which can be shed post-vaccination (causing other horses to seroconvert); therefore, horses should be isolated for at least 21 days after administration of the vaccine.

\section{Many states have adopted} statutory language governing the prevention and control of EVA. For example, the State of Utah requires all stallions used for breeding entering Utah be tested

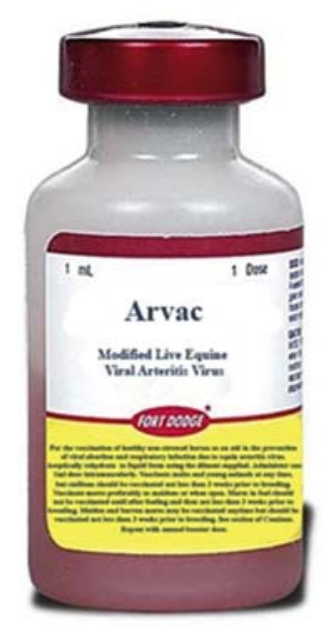
for Equine Viral Arteritis by an accredited veterinarian within 30 days prior to entry by rule (R58-23). Exceptions to the above are stallions that have proof of negative EVA status prior to vaccination and proof of subsequent yearly vaccination. No EVA carrier stallion used for breeding purposes shall be permitted to enter Utah without a prior permit from the State Veterinarian. No semen from a carrier stallion shall be permitted to enter into Utah without a prior permit from the 
State Veterinarian. All EVA Carrier Stallions, used for breeding purposes, imported into Utah shall be taken directly to an approved facility and shall remain on said facility until permission from the State Veterinarian is obtained to move the animal to another approved facility. All semen from an EVA Carrier Stallion imported into Utah shall be shipped directly to an approved facility and shall remain on said facility until inseminated, transported to another approved facility and/or disposed of.

All stallions used for breeding purposes identified as EVA positive shall have their semen tested by an accredited veterinarian at an approved laboratory prior to breeding. All carrier stallions used for breeding purposes shall be housed and maintained at an approved facility until permission from the State Veterinarian is given to move the stallion to another approved facility. All EVA infected semen shall only be collected, handled, evaluated, received, packaged and/or administered at an approved facility.

All equids, including but not limited to stallions, mares and geldings, on approved facilities shall be vaccinated for EVA no less than 21 days before the start of breeding season or no less than 21 days before arriving at an approved facility. Mares being bred to a carrier stallion, or inseminated with semen from a carrier stallion, shall remain on the approved facility for a minimum of 21 days after the initial breeding date. Adequate biosecurity precautions shall be in place during the breeding season. The adequacy of biosecurity may be monitored periodically by the Utah Department of Agriculture and Food.
The private veterinary practitioner immediately upon receiving a positive laboratory report on EVA shall report all EVA positive equids to the State Veterinarian. All EVA positive test results processed at a state owned laboratory shall be immediately reported to the State Veterinarian. The State Veterinarian may require testing of any stallion suspected of being exposed to EAV.

\section{Summary}

Equine viral arteritis is now recognized as an important health threat in the equine industry, particularly in animals used for breeding. Through appropriate control and prevention procedures, such as serological testing and vaccination, the risk of EVA can be minimized. An important strategy for controlling EVA is prevention of the persistently infected carrier state in stallions. Because of the regulatory aspect of EVA, it is imperative that horse owners contact their respective states' regulatory authority for information regarding rules in place that govern the control of EVA.

\section{Additional Information}

The United States Department of Agriculture (USDA), Animal and Plant Health Inspection Service (APHIS), Veterinary Services (VS) has several information resources about EVA that are available online at:

http://www.aphis.usda.gov/animal_health/animal_di seases/eva/

The American Association of Equine Practitioners (AAEP) has a fact sheet on EVA that can be accessed online at: http://www.aaep.org/eva.htm

Utah State University is committed to providing an environment free from harassment and other forms of illegal discrimination based on race, color, religion, sex, national origin, age (40 and older), disability, and veteran's status. USU's policy also prohibits discrimination on the basis of sexual orientation in employment and academic related practices and decisions.

Utah State University employees and students cannot, because of race, color, religion, sex, national origin, age, disability, or veteran's status, refuse to hire; discharge; promote; demote; terminate; discriminate in compensation; or discriminate regarding terms, privileges, or conditions of employment, against any person otherwise qualified. Employees and students also cannot discriminate in the classroom, residence halls, or in on/off campus, USU-sponsored events and activities.

This publication is issued in furtherance of Cooperative Extension work, acts of May 8 and June 30, 1914, in cooperation with the U.S. Department of Agriculture, Kenneth L. White, Vice President for Extension and Agriculture, Utah State University. 